\title{
ECOLOGÍA DEL COMPORTAMIENTO HUMANO: LAS CONTRADICCIONES TRAS EL MENSAJE DE CRISIS AMBIENTAL
}

\author{
Fabiola Cifuentes-Ávila ${ }^{1}$, Rolando Díaz-Fuentes ${ }^{2}$, Sonia Osses-Bustingorry ${ }^{3}$
}

Resumen: El artículo aporta a la discusión respecto del entendimiento actual del comportamiento ecológico del ser humano desde una relación de dominación utilitaria del ambiente, en contraste a su comprensión desde la perspectiva ecológica, la cual responde a un modelo natural que es parte de las relaciones de su funcionamiento en el ecosistema. $\mathrm{Al}$ respecto, se pone en discusión el paradigma de crisis ambiental, en el cual dicho comportamiento supone un grave problema de la sociedad. Desde los autores, la problemática estaría ligada íntimamente al sistema productivo y económico que promueve la artificialización del ser humano, creando falsas necesidades que, mediante su consumo, aseguran la sostenibilidad del modelo económico. Se plantea la reflexión crítica desde la educación, hacia una nueva comprensión del comportamiento humano cuya perspectiva ecológica impulse nuevas relaciones ecosistémicas, fundamentalmente basadas en relaciones de colaboración.

Palabras clave: comportamiento ecológico, necesidades egocéntricas, consumismo, crisis ambiental

\section{Ecology of human behavior: contradictions behind the message of environmental crisis}

\begin{abstract}
The article contributes to the discussion about the current understanding of the ecological behavior of the human being from a relation of utilitarian domination of the environment in contrast to its understanding from the ecological perspective, which responds to a natural model that is part of the relations of its functioning in the ecosystem. In this regard, the paradigm of environmental crisis is discussed, in which such behavior is a serious problem of society. From the authors, the problem would be closely linked to the productive and economic system that promotes the artificialization of the human being, creating false needs that through their consumption ensure the sustainability of the economic model. Critical reflection is proposed from education, towards a new understanding of human behavior whose ecological perspective drives new ecosystemic relations, fundamentally based on collaborative relationships.
\end{abstract}

Key words: ecological behavior, egocentric needs, consumerism, environmental crisis

\section{Ecologia do comportamento humano: as contradiçóes por trás da mensagem de crise ambiental}

Resumo: $\mathrm{O}$ artigo traz à discussão a compreensão atual do comportamento ecológico do ser humano, a partir de uma relação de dominaçáo utilitária do ambiente em contraste com a sua compreensão do ponto de vista ecológico, a qual responde a um modelo natural que faz parte de relaçôes de seu funcionamento no ecossistema. A este respeito, é colocado em discussão o paradigma de crise ambiental, em que tal comportamento supóe um problema grave da sociedade. Para os autores, a questão está intimamente ligada ao sistema produtivo e econômico que promove a artificialização do ser humano, criando falsas necessidades que, por meio de seu consumo, garantam a sustentabilidade do modelo econômico. É realizada uma reflexão crítica a partir da educação, no sentido de uma nova compreensão do comportamento humano, cuja perspectiva ecológica impulsiona novas relaçôes ecossistêmicas baseadas na cooperação.

Palavras-chave: comportamento ecológico, necessidades egocêntricas, consumismo, crise ambiental

\footnotetext{
${ }^{1}$ Universidad de La Frontera, Temuco, Chile. Correspondencia: fabiolacif@gmail.com

${ }^{2}$ Departamento de Educación, Facultad de Educación, Ciencias Sociales y Humanidades, Universidad de La Frontera, Temuco, Chile. Comité Pro-Defensa de la Fauna y Flora (CODEFF), Chile

${ }^{3}$ Departameno de Educación, Facultad de Educación, Ciencias Sociales y Humanidades, Universidad de La Frontera, Temuco, Chile.
} 


\section{Introducción}

En la actualidad, el comportamiento humano en relación al ambiente es entendido desde una mirada antropocéntrica, lo cual supone un grave problema que atenta contra los ecosistemas. En este sentido, diversos autores a nivel internacional concuerdan en que dicho comportamiento sería la causa principal de los problemas ambientales que enfrenta el planeta, cuyos efectos han derivado en una crisis ambiental global(1-5).

$\mathrm{Al}$ respecto, el sistema económico predominante ha promovido un mensaje de crisis ambiental mediante el cual va creando falsas necesidades sobre la base de la capitalización y mercantilización de todo lo existente, incluido el propio ambiente, asegurando de esta forma la sostenibilidad del modelo económico(6). Como señalan Barkin, Fuente y Tagle, desde esta postura antropocéntrica se privilegia el crecimiento económico por sobre el cuidado y respeto del ambiente(7), lo que influye directamente en las relaciones entre el ser humano y este, llevando al hombre a concebirse como agente externo al ecosistema y olvidando su relación en interdependencia con el resto de los organismos.

En contraste a lo anterior, este artículo asume una perspectiva ecológica, en la cual el ser humano sienta que es parte constituyente e interactuante del ecosistema y, por tanto, su "comportamiento ecológico" sea una comprensión de las relaciones funcionales del ecosistema global, el cual responde a un modelo natural de predación que incluye los factores de competencia y colaboración(8). Al respecto, destacan los aportes realizados por $\mathrm{Ca}$ raballo y Barros, quienes afirman que, por nefasto que el comportamiento parezca, es parte de las relaciones necesarias del organismo en el ecosistema(9) y, por lo tanto, no sería un problema, sino un proceso natural.

El propósito de este artículo es aportar a la discusión respecto de la comprensión del comportamiento ecológico del ser humano y las contradicciones éticas asociadas al mensaje de crisis ambiental que promueve el modelo económico neoliberal, con el fin de reflexionar respecto de nuestra relación con el ambiente, entendiéndose a éste como un conjunto de organismos interde- pendientes. Se cree que, mediante esta comprensión, será posible a las sociedades ocuparse de los deberes y responsabilidades que deben asumirse con la naturaleza, el resto de los seres vivos y las futuras generaciones $(10,11)$, contribuyendo de esta forma al desarrollo ético del saber.

\section{Comportamiento ecológico y ser humano}

El comportamiento del ser humano ha demostrado tener un impacto sobre el ambiente(12). Evidencia de ello es la degradación y contaminación ambiental que afecta al planeta en la actualidad, constituyendo una de las principales preocupaciones mundiales(7).

En todo el globo, los antecedentes confirman la existencia de un problema. La contaminación de las aguas dulces, especialmente en ríos de América Latina, África y Asia, ha empeorado a tal punto que cerca de un tercio de la biodiversidad de sus ecosistemas ya se ha perdido $(13,14)$. Por su parte, la sobreexplotación de especies ha llevado a la extinción del $58 \%$ de los peces y a la pérdida del $38 \%$ de la biodiversidad terrestre desde los años setenta a una tasa de $1,1 \%$ en promedio(15). Las concentraciones de contaminantes del aire en las grandes ciudades del mundo sobrepasan los límites declarados como seguros por la Organización Mundial de la Salud y se proyecta que el número de muertes por exposición a partículas suspendidas hacia el año 2050 alcanzará los 3.6 millones de personas al año en el mundo(13). Por su parte, en las ciudades de América Latina y el Caribe una de las principales fuentes de contaminación son los residuos sólidos, donde solo durante el año 2010 se generaron 436.000 toneladas, de las cuales cerca del $50 \%$ ha terminado en rellenos sanitarios y el resto desechado en vertederos(16).

En Chile la situación es similar. La contaminación atmosférica de las zonas urbanas es permanentemente alta, la escasa agua disponible está contaminada y se está perdiendo hábitat; las emisiones de gases de efecto invernadero se han incrementado en un $23 \%$ entre 2000 y 2010, lo cual es considerado uno de los porcentajes más altos entre los países miembros de la Organización para la Cooperación y Desarrollo Económico (OCDE), y se ha calculado que, para el caso de Chile, exista una mortalidad de más de 4.000 personas al año 
debido a enfermedades cardiopulmonares asociadas a la exposición a material particulado(17). Por su parte, las plantaciones, la expansión de zonas agrícolas y urbanas, la tala ilegal y los incendios forestales han provocado la pérdida de una gran parte de vegetación nativa en el país y la sobreexplotación de los ecosistemas marinos ha llevado a una situación de extinción a poblaciones de peces como el jurel y la anchoa(17).

A lo anterior se suma el aumento que ha experimentado el manejo irresponsable de residuos sólidos, alcanzando una generación estimada de 6,5 millones de toneladas de residuos municipales solo hacia el año 2009(18). Al respecto, cabe señalar que en la actualidad más del 95\% de los residuos recogidos se continúan almacenando en vertederos(17), método de eliminación de desechos que ya en la década de los noventa Odum y Sarmiento declaraban como inadmisible(8), pero que en nuestros días es parte de las acciones que en Chile aún se realizan.

Es en este contexto, las personas se desarrollan en continua interrelación con otros y su ambiente, evidenciando un determinado comportamiento, el cual, desde la mirada antropocéntrica, es presentado como un problema que atenta contra los ecosistemas, generando el paradigma de crisis ambiental(19). Al respecto, en las últimas semanas se han venido presentado, en sitios no especializados, discusiones en torno a la responsabilidad del ser humano de actuar frente a la crisis existente. Por un lado, autores afirman que no necesitamos salvar especies en peligro de extinción, puesto que la misma es parte de la evolución. Por otro lado, las réplicas señalan que sí debemos proteger la biodiversidad, debido a que la escala actual de extinción no tiene precedentes(20).

Desde una perspectiva ecológica, el ser humano, al igual que todo organismo, constituye parte integral del ecosistema(9) y, por tanto, su "comportamiento ecológico" obedece a su funcionamiento natural en una red de interrelaciones, dependiendo del ambiente y sus recursos para subsistir y prosperar $(21,22)$.

Según sus necesidades y movido a actuar en favor de su sobrevivencia, el ser humano demanda del ambiente recursos y energía; se comporta como un competidor y depredador con el fin de obtener alimentos, combustibles, fibras, refugio, reproducción, agua, aire, suelo y otros que considera necesarios(8). Estos son utilizados, transformados y devueltos como desechos que impactan el ambiente, pero que contribuyen al flujo de materia y energía en el ecosistema(23). En este sentido, el "comportamiento ecológico", por perjudicial que parezca, es parte natural del ecosistema(9).

El ser humano, por su condición pensante, presenta necesidades que van desde las fisiológicas indispensables para la subsistencia - hasta aquellas de reconocimiento social y autorrealización; a diferencia de otros organismos, es un "animal necesitado" que, excepto en breves periodos de tiempo, nunca alcanza un estado de completa satisfacción; sus necesidades en cuanto ser social no tienen límite y, al saciar cualquiera de ellas, surge en su lugar una nueva y superior, necesitando una alta valoración de sí mismo, la estima de otros, prestigio, estatus, fama y gloria $(24,25)$. Son precisamente estas necesidades, altamente egocéntricas, las que, a nuestro entender, distinguen al hombre del resto de los organismos, llevándolo a competir no tan solo por recursos, sino por identidad y prestigio social(26).

Por su parte, la permanente insatisfacción del ser humano le ha llevado a la búsqueda de nuevas formas de relacionarse con el ambiente, promoviendo modificaciones en el sistema productivo y económico basadas en la dominación y el manejo utilitario de la naturaleza(6).

\section{Modelo económico y crisis ambiental}

El actual modelo económico se caracteriza por la extracción de recursos naturales y su capitalización en dinero, lo que genera ganancias y un mayor nivel de consumo que impulsa y sostiene al sistema(27).

Respondiendo a las necesidades egocéntricas del ser humano, el modelo crea falsas necesidades que luego dice satisfacer, anima a comprar cosas innecesarias, ofrece soluciones a problemas inexistentes e impulsa elementos cargados de significancia simbólica que aumentan el estatus social(28). Por lo tanto, todo lo existente tiene un potencial de mercantilización que puede ser convertido en 
objeto de valor dispuesto para ser apropiado, explotado y aprovechado para dinamizar aún más el modelo económico neoliberal(6).

Bajo esta lógica, el modelo económico neoliberal ha promovido un mensaje de crisis, en el cual el comportamiento del hombre pasa a ser un problema que atenta contra los ecosistemas(7), desnaturalizando la competencia, la degradación y la contaminación. Dicho mensaje permite a este modelo crear nuevas necesidades, entre ellas, vivir en un ambiente sano, el cual es presentado como bien de consumo, pudiéndose acceder a el con dinero, principalmente mediante servicios ecosistémicos, asignando un valor monetario a la naturaleza, lo que refuerza el paradigma de mercantilización. Prueba de ello es el mensaje del PNUD en la XI Convención Internacional sobre Medio Ambiente y Desarrollo realizada en Cuba, en julio de 2017, que menciona: "Nature Count\$".

En este sentido, nos hablan de crisis y asumimos una mirada antropocéntrica, excluyéndonos del ecosistema del cual somos parte, llevándonos a pensar desde afuera para salvaguardarnos como especie; ¿salvaguardarnos de qué? ¿De lo natural? ¿Del deterioro? ¿Del desgaste? ¿De la muerte? Es necesario reflexionar sobre aquello que hemos olvidado: somos parte de un modelo de consumo de energía, crecimiento y mortalidad que es inherente a la vida; parte integral del ecosistema en que dependemos del ambiente para subsistir, le impactamos y somos impactados. "Nuestro comportamiento ecológico no es un problema", es parte de las interrelaciones que incluye competencia y depredación, que aseguran la subsistencia, flujo de materia y energía en el ecosistema, y donde la eliminación de desechos es parte del proceso. He aquí, una nueva necesidad, que no atiende a la mercantilización sino a la conciencia que como especie nos caracteriza; que, empodera- dos de nuestro rol en el ecosistema de competir, depredar y contaminar, asumamos el llamado a expandirnos hacia nuevas relaciones ecológicas, que implican colaboración, mutualismo o comensalismo, las cuales puedan convertirse en bases de nuevas economías y en pilares fundamentales para un cambio de paradigma que nos lleve a comprender que en el ecosistema somos interdependientes con el resto de organismos que lo conformamos, lo cual ha de impulsarnos por el camino de la solidaridad que permita a la especie humana una real transformación.

\section{Conclusiones}

La reflexión en torno al entendimiento actual del comportamiento ecológico del ser humano y de la crisis ambiental, puede llevarnos hacia una nueva comprensión respecto de nuestras relaciones de interdependencia con la naturaleza.

Al respecto, conscientes de la importancia de la educación como camino para la transformación social, se recomienda revisar los sistemas educativos que van replicando estos modelos de desarrollo fundados en la competencia.

Finalmente, transitar hacia un currículo basado en elementos de solidaridad y colaboración permitirá ocuparnos como sociedad de las responsabilidades y deberes que tenemos con la naturaleza, el resto de organismos y con nosotros mismos.

\section{Agradecimientos}

A la Comisión Nacional de Investigación en Ciencia y Tecnología, CONICYT, por el financiamiento del Programa de Doctorado en Ciencias de la Educación de la Universidad de La Frontera, marco en que se inserta el presente artículo.

\section{Referencias}

1. Covarrubias F, Ojeda A, Cruz M. La sustentabilidad ambiental como sustentabilidad del régimen capitalista. Ciencia Ergo 2011; 18(1): 95-101.

2. Moyano E, Cornejo F, Gallardo I. Creencias y conductas ambientales, liberalismo económico y felicidad. Acta Colombiana de Psicología 2011; 14(2): 69-77.

3. Touguinha S, Pato C. Valores personales, creencias ambientales ecocéntricas y comportamiento ecológico de trabajadores brasileños: el caso del ministerio público del Distrito Federal y territorios. Quaderns de Psicología 2011; 13(1): 35-45. 
4. Moyano E, Palomo G, Moyano P. Creencias ambientales e ideología en población chilena. Universum 2015; 30(2): 219-236.

5. Palacios J, Bustos M, Soler L. Factores socioculturales vinculados al comportamiento proambiental en jóvenes. Revista de Psicología 2015; 24(1): 1-6.

6. Gudynas E. Imágenes, ideas y conceptos sobre la naturaleza en América Latina. Cultura y Naturaleza 2010; 267-292.

7. Barkin D, Fuente M, Tagle D. En el crecimiento económico, la crisis ambiental y el marxismo. Debates y perspectivas epistemológicas. Pensar Epistemología y Ciencias Sociales 2011; 6: 15-30.

8. Odum E, Sarmiento F. Ecología: el puente entre ciencia y sociedad. México D.F.: Mc Graw-Hill Interamericana; 1998.

9. Caraballo P, Barros N. Ecología y bienestar humano. Revista Colombiana de ciencia animal 2011; 3 (1): 141-147.

10. Lecaros J. La ética medio ambiental: principios y valores para una ciudadanía responsable en la sociedad global. Acta Bioethica 2013; 19(2): 177-188.

11. Lolas F. Bioética: interdisciplinariedad, interculturalidad, interdiscursividad. Acta Bioethica 2017; 23(2): 211-212.

12. Miranda L. Cultura ambiental: un estudio desde las dimensiones de valor, creencias, actitudes y comportamientos ambientales. Producción Más Limpia 2013; 8(2): 94-105.

13. Organización para la Cooperación y el Desarrollo Económico. Perspectivas ambientales de la OCDE hacia $2050 ; 2012$. Disponible en: https://www.oecd.org/env/indicators-modelling-outlooks/49884278.pdf. (último acceso 22 de noviembre 2017).

14. Organización de las Naciones Unidas para la Educación, la Ciencia y la Cultura. Agua y empleo. Informe de las Naciones Unidas sobre el desarrollo de los recursos hidricos en el Mundo; 2016. Disponible en: http://unesdoc.unesco.org/ images/0024/002441/244103s.pdf. (último acceso 22 de noviembre 2017).

15. World Wildlife Fund. Planeta Vivo. Informe 2016: Riesgo y resiliencia en una nueva era; 2016. Disponible en: http:// d2ouvy59p0dg6k.cloudfront.net/downloads/informe_planeta_vivo_2016_2.pdf. (último acceso 22 de noviembre 2017).

16. Comisión Económica para América Latina y el Caribe. El desafío de la sostenibilidad ambiental en América Latina y el Caribe; 2015. Disponible en: http://repositorio.cepal.org/bitstream/handle/11362/37791/LCM23_es. pdf. (último acceso 06 de diciembre 2017).

17. Comisión Económica para América Latina y el Caribe. Evaluaciones del desempeño ambiental, Chile: aspectos destacados; 2016. Disponible en: http://repositorio.cepal.org/bitstream/handle/11362/40308/S1600413_es.pdf. (último acceso 22 de noviembre 2017).

18. Comisión Nacional del Medio Ambiente. Balance y Perspectivas de la Educación Ambiental en Chile e Iberoamérica; 2010. Disponible en: http://educacionysustentabilidad.tecsuma.cl/wp-content/uploads/2011/11/ EA-CHILE-01-BalanceEd-Amb-Chile-Latin-America-2010.pdf. (último acceso 06 de diciembre 2017).

19. Mora W. Educación ambiental y educación para el desarrollo sostenible ante la crisis planetaria: demandas a los procesos formativos del profesorado. Tecné, Episteme y Didaxis 2009; 26: 7-35.

20. Pire A. We do not need to save endangered species. Extinction is part of evolution. USA: The Washington Post; 2017. Disponible en: https:/www.washingtonpost.com/outlook/we-dont-need-to-save-endangered-species-extinction-is-part-ofevolution/2017/11/21/57fc5658-cdb4-11e7-a1a3-0d1e45a6de3d_story.html?tid=a_inl\&utm_term=.e07200b04433. (último acceso 20 de diciembre 2017).

21. Margalef R. Teoría de los sistemas ecológicos. México D.F.: Alfa Omega; 2002.

22. Armenteras D, González TM, Vergara LK, et al. Revisión del concepto de ecosistema como "unidad de la naturaleza" 80 años después de su formulación. Ecosistemas 2016; 25(1): 83-89.

23. Isaza J, Campos D. Ecología: una mirada desde los sistemas dinámicos. Bogotá: Editorial Pontificia Universidad Javeriana; 2006.

24. Maslow A. Motivación y personalidad. Madrid: Ediciones Díaz de Santos S.A; 1991.

25. Baudrillard J. La sociedad del consumo, sus mitos, sus estructuras. Madrid: Siglo XXI; 2009.

26. Rodríguez S. Consumismo y Sociedad: una visión crítica del Homo Consumens. Nómadas 2012; 34.

27. Giddens A. El capitalismo y la moderna teoría social. Barcelona: Editorial Labor; 1998.

28. Buckingham D. La infancia materialista: Crecer en la cultura consumista. Madrid: Morata; 2013.

Recibido: 21 de septiembre de 2017

Aceptado: 22 de diciembre de 2017 